\title{
Gut-Hepatic Relationship: From Disorders of the Gut Microbiota to Hepatocellular Carcinoma
}

\author{
Ilkham Murkamilov', Ibragim Sabirov², Victor Fomin ${ }^{3}$, Furkat Yusupov ${ }^{4}$ and Ziabidin \\ Aidarov $^{1}$ \\ ${ }^{1}$ I K Akhunbaev Kyrgyz State Medical Academy, Kyrgyzstan \\ ${ }^{2}$ Kyrgyz Russian Slavic University, Kyrgyzstan \\ ${ }^{3}$ I M Sechenov First Moscow State Medical University, Russian Federation \\ ${ }^{4}$ Osh State University, Kyrgyzstan \\ *Corresponding author: Ilkham Murkamilov, I.K. Akhunbaev Kyrgyz State Medical Academy, Bishkek 720020, Kyrgyzstan. \\ To Cite This Article: Ilkham Murkamilov, Ibragim Sabirov, Victor Fomin, Furkat Yusupov, Ziabidin Aidarov. Gut-Hepatic Relationship: From \\ Disorders of the Gut Microbiota to Hepatocellular Carcinoma. Am J Biomed Sci \& Res. 2021 - 11(6). AJBSR.MS.ID.001681. DOI: 10.34297/ \\ AJBSR.2021.11.001681
}

Received: 眥 January 06, 2021; Published: 跙 February 03, 2021

\begin{abstract}
Enterohepatic relationships reflect the integrity of homeostasis regulation in the human body. Anatomical and physiological connection through the portal vein of the intestine and liver ensures the transport of products derived from the intestine directly to the liver and through the liver ensures the connection of bile and the secretion of antibodies into the intestine. However, impairment of intestinal barrier integrity, endotoxemia, increase of lipopolysaccharides in hepatocytes, Kupffer and stellate cells with increasing content of pro-inflammatory cytokines and active oxygen forms creates inflammatory environment with sensitization of liver cells to injury and profibrotic processes. The goals of experimental and clinical research in this course are multifaceted, ranging from regulating human metabolism, immune and inflammatory reactions to preventing carcinogenesis, inhibition of liver cancer progression and improving the efficiency of liver cancer treatment. Here we will discuss epidemiological issues of hepatocellular carcinoma, the role of intestinal microbiota when enterohepatic relationships are impaired, and the intestinal-associated mechanisms of carcinogenesis in this form of liver cancer.
\end{abstract}

Keywords: Gut microbiota, Enterohepatic relationships, Hepatocellular carcinoma, Risk factors, Dysbiosis, Lipopolysaccharides, Pro-inflammatory cytokines, Bile acids, Short-chain bile acids, Choline deficiency, Ethanol, Acetaldehyde

\section{Introduction}

One of the leading causes of death worldwide is hepatocellular carcinoma. Research in recent years shows the important role of enterohepatic relationships in the pathophysiological mechanism responsible for the development and progression of HCC. The intestinal microflora is a positive factor of human homeostasis and immune reactions due to precise control and immunosensory ability to distinguish between commensal and pathogenic bacteria. Reciprocal enterohepatic relationships are established by means of a portal vein, which provides transport of products derived from the intestine directly to the liver and through the liver provides bile feedback and antibody secretion into the intestine. Liver products primarily affect the composition of the gut microbiome and the integrity of the intestinal barrier, while intestinal factors regulate the synthesis of bile acids, the metabolism of glucose and lipids in the liver.

Pro-inflammatory changes in the liver and intestines will mediate the formation of fibrosis, cirrhosis and, eventually, HCC. The exact contribution of microbiome to liver disease may vary depending on the etiology. Gut-associated mechanisms of carcinogenesis in HCC develop due to damage of the intestinal barrier integrity in the deficit of bile acids, short-chain bile acids and choline. Later, with the excess growth of microbes in the intestine, endotoxemia is progressed, inflammatory environment is formed with an increase in the content of lipopolysaccharides associated 
with specific TLR4-receptors of hepatocytes, Kupffer and stellate cells, with subsequent release of pro-inflammatory cytokines and active oxygen forms. Ethanol also plays an essential role in the progression of HCC, its metabolism product - acetataldehyde, as well as increase of intestinal bacterial metabolite, deoxycholic acid. Research focused on understanding the functioning of the enterohepatic axis is aimed at improving the treatment of liver diseases, including hepatocellular carcinoma. The data from experimental studies on animals allow to find out the etiological mechanisms of HCC progression, however, well planned, largescale clinical trials covering etiology of various liver diseases, as well as ethnicity of patients are required in clinical practice for effective interpretation and application of the results obtained in the experiment.

\section{Hepatocellular Carcinoma: Epidemiology Issues}

Hepatocellular carcinoma (HCC) is the most common primary carcinoma of the liver. Liver cancer is the sixth most frequently diagnosed and the fourth in the mortality rate due to cancer in the world after lung cancer, colorectal cancer, and stomach cancer [1]. HCC is one of many types of tumors that occur against the background of chronic inflammation. HCC, which accounts for $90 \%$ of all primary liver cancers [2,3].

According to Singal AG and co-authors (2020), the prevalence of HCC is not homogenous around the world due to different prevalence of major risk factors. It is estimated that $72 \%$ of progression cases occur in Asia (over 50\% in China), 10\% in Europe, 7.8\% in Africa, 5.1\% in North America, 4.6\% in Latin America and $0.5 \%$ in Oceania [1]. Singal AG et al. (2020), highlighting new trends in the epidemiology and epidemiological surveillance of HCC, note that the highest estimated age standardized incidence rate (ASIR) for liver cancer in the world in 2018 per 100,000 people is found in East Asia (17.7), with the highest ASIR in the region and the world recorded in Mongolia (93.4), followed by Southeast Asia (13.3) and Africa (8.4), with Egypt (32.2) and Gambia (23.9) having the highest ASIR in Africa. The lowest ASIR was observed in South and Central Asia (2.5), followed by Central and Eastern Europe and Western Asia (approximately 4.0) [1]. Moreover, age standardized mortality rates (ASMR) due to HCC in 2018 are also the highest in East Asia (16.0) and North Africa (13.9), followed by Southeast Asia (13.2). The lowest ASMR is observed in South and Central Asia (2.3), followed by Central, Northern and Eastern Europe and Western Asia (about 3.8-4.0). Mongolia and Egypt have the highest ASMRs, and the lowest ASMRs are in Morocco and Nepal, the countries with low ASIR [1]. Liver cancer is a tumor with high mortality, with most cases being detected at late stages and the morbidity-to-mortality ratio approaching 1 . It is important to note that HCC is the result of many etiological factors, such as viral infections of hepatitis $\mathrm{B}$ and $\mathrm{C}$, non-alcoholic fatty liver disease (NAFLD) and non-alcoholic steatohepatitis (NASH), alcoholic liver disease (ALD), autoimmune hepatitis and several genetic disorders. Hepatitis B virus (HBV) is the main cause of liver cancer and mortality in the world (33\%), followed by alcohol (30\%), hepatitis C virus (HCV) (21\%) and other causes (16\%) [1]. The main risk factors for HCC, including infection with hepatitis B virus (HBV) or hepatitis C virus (HCV), lead to the formation and progression of liver cirrhosis (LC), which is observed in $80-90 \%$ of patients with HCC. The five-year cumulative risk of HCC progression in patients with liver cancer varies from 5 to $30 \%$ [2]. With the introduction of vaccination programs against hepatitis $\mathrm{B}$ and treatment of hepatitis $\mathrm{C}$ worldwide, the epidemiology of HCC shifts from a disease in which viral hepatitis prevails to the hepatic component of metabolic syndrome - NAFLD [3], where about $10-30 \%$ of cases of NAFLD progress to liver cancer. More than 65 million Americans are affected by NAFLD, which costs 103 billion dollars a year in the United States itself [1]. There are data on the influence of lipid reduction strategy, including ezetimibe, and its combination with other hypolipidemic agents on atherogenic dyslipidemia, glycemic profile, and morphological changes in liver steatosis [5].

The progression of HCC in NASH occurs in the absence of LC [6] in more than $25 \%$, which is significantly higher than the frequency observed in other liver diseases [7]. HCC has a strong prevalence of men in morbidity and mortality, with a ratio of men to women exceeding 2.5 for both epidemiological indicators [8]. It is believed that this differential distribution by gender is due to the clustering of risk factors among men, as well as the potential impact of androgens on the risk of HCC. There are also essential environmental risk factors for HCC. For example, in some parts of Africa and Asia, an important factor for the progression of HCC is the consumption of Aflatoxin B1 with food, which occurs due to fungal contamination of staple foods. The impact of Aflatoxin B1 is closely related to the mutations of TR53 (codon 249) and the progression of HCC in people infected with HBV [9]. Several epidemiological studies have identified an increased risk of HCC progression among smokers, with meta-analysis reporting an adjusted OR 1.5 (95\% of CI 1.37-1.67) compared to non-smokers [10]. Study of HCC prevention in the general population and in patients with chronic liver disease, coffee consumption, aspirin intake and metformin treatment consistently reduce the incidence of HCC in diabetics [11-13].

\section{Gut Microbiota and Its Role When Enterohepatic Relationships are Impaired}

The intestines and liver interact through close bi-directional connections through the bile paths, portal vein and systemic circulation. The liver binds to the intestine, releasing bile acids and many biologically active mediators in the biliary tract and the systemic circulation. In the intestine, the host and microbes metabolize endogenous (bile acids, amino acids), as well as exogenous substrates (against diet and environmental influences), 
which products are transported to the liver through the portal vein and affect liver function $[14,15]$. An important factor in liver injury is disturbance of the gut microbiota (GM) system. The human gastrointestinal tract (GI tract) colonizes a large number of microbial microflora, forms a system of metabolism of the microbiota with the macroorganism, participating in various metabolic processes of the body, and plays an essential role in the human immune response. More than 2,000 different types of bacteria live in the intestines, and their number exceeds 100 trillion microorganisms, which is 10 times more than the total number of human cells [16]. Gut microbiome, which refers to the collective genomes of all microorganisms that make up the gut microflora, contains 150 times more genes than the human genome [17]. The number of different microorganisms is gradually increased during the intestine. This can be explained by the presence of a more aggressive environment in the upper parts of the intestine due to the incoming acidic content of the stomach, the action of digestive enzymes, rapid progression of chyme. The density of microbes increases from proximal to distal end of the intestine and includes a biomass of $1.5-2.0 \mathrm{~kg}$, which is dominated by strictly anaerobic bacteria [18]. Aerobia predominating in the small intestine, as they progress down the GI tract, are replaced by facultative and then strict anaerobes. These microorganisms are collectively referred to as gut microbiota (IM), which consists of commensals, beneficial bacteria, and opportunistic pathogenic bacteria and pathogenic bacteria in a complex and dense microenvironment $[19,20]$.

The GI tract, which functions as an effective barrier against endotoxin and intestinal bacteria, can protect the body [21]. In addition, the liver also plays an essential physiological role in detoxification of lipopolysaccharides (LPS) and hepatocytes participate in intestinal endotoxin clearance [22]. The process of endotoxin degradation can be disrupted if the liver is damaged, and the increase in endotoxin, in turn, exacerbates the damage to the liver. GM is involved in the metabolism of bile acids (BA), the synthesis of vitamins, the assimilation of complex polysaccharides and the production of short-chain BA (SCBA) [23]. SCBAs are a vital energy source for enterocytes, which are an integral part of maintaining intestinal barrier integrity. In addition, GM is also involved in the development of local and congenital immunity, providing protection not only against pathogenic invasion but also against systemic infection [24]. Thus, the gut microbiome is now considered an organ system that is central to maintaining metabolic and immunological homeostasis. It is involved in the production of compounds necessary for life, for the metabolism of many compounds that enter the body, and on the one hand, stimulates innate and adaptive immunity, and on the other hand, softens the immune response. Changes in the composition of bacteria (and other microbes) in the intestines in a state known as dysbacteriosis are associated with a growing and significant number of diseases characterized by chronic inflammation affecting various organ systems, including the liver [24- 29].

\subsection{Gut-Associated Carcinogenesis Mechanisms}

in

Hepatocellular Carcinoma

On the experimental model of dysbiosis the link between intestinal colonization of $\mathrm{H}$. hepaticus and development of HCC was proposed [30-32]. Huang $Y$ et al. reported the presence of Helicobacter ssp DNA in liver biopsies from patients with HCC while being virtually absent from control samples [32]. Of note, the representatives of this kind are known to promote tumor development by activating the transmission of intracellular NF$\mathrm{kB}$ and WNT signals that regulate cell differentiation and the development of malignant tumors, as well as suppressing antitumor immunity, which may play a potential role in the development of HCC [30,33]. On the other hand, Rocha et al. (2005) confirmed an association between the presence of Helicobacter ssp DNA and liver cirrhosis, but not a correlation between the presence of Helicobacter ssp DNA and HCC [34]. However, there is evidence of a correlation between the presence of helicobacter DNA in patients' stool and development of HCC in patients with viral hepatitis [35]. Elevated intestinal level of Clostridium microbial species has been found in experimental models of HCC induced by obesity in mice [36,37], but clinical studies involving patients with HCC found excessive Escherichia coli growth [38]. Patients with liver cirrhosis and liver cancer frequently develop an intestinal dysbiosis which is also found in mice after DEN-administration, which show an increase growth rate of the E. coli and Atopobium cluster - gram-positive anaerobic microorganisms belonging to the Coriobacteriaceae family, which are associated with the development of bacterial vaginosis and inflammatory diseases of the small pelvis, while the percentages of benign bacteria (Lactobacillus group, Bifidobacterium group, and Enterococcus group) are significantly decreased [39].

Etiological factors of non-viral HCC lead to steatosis of the liver accompanied by oxidative stress, endoplasmic reticulum stress, intestinal dysbiosis and inflammation, which contributes to the final manifestation of cancer [40]. Yu LX et al. (2010) demonstrated that experimental depletion of host microflora suppresses tumor formation in a toxic model of hepatocarcinogenesis. Treatment of rats with polymyxin B and neomycin, which are bactericidal for most enteric gram-negative organisms, after DEN administration injection significantly reduced the number and size of HCC nodules [41]. These data were corroborated by findings of Dapito DH and colleagues demonstrating that gut sterilization protects from development of liver cancer when mice were subjected to a combination of diethylnitrosamine (DEN) and hepatotoxin carbon tetrachloride ( $\mathrm{CCl} 4$ ), a model that features several characteristics of the cirrhotic environment of chronically injured livers in which HCC mostly arises, namely chronic injury, inflammation, fibrogenesis, and increased of endotoxin levels [42]. 
The antibiotic treatment led to a reduction of tumor number and size compared with mice that did not receive antibiotics. Moreover, mice that were grown in specific germ-free conditions demonstrated fewer and smaller tumors in this model compared with mice that were grown under non-germ free (SPF) conditions [42]. Human HCC is often associated with chronic inflammation of the liver and liver cirrhosis, pathophysiological processes that are the result of chronic viral infection, metabolic disorders or exposure to chemical toxins that can develop against the background of inflammatory environment in patients with advanced liver disease. Experimental data from rodent models as well as robust clinical data suggest a role of inflammation in the pathophysiology of HCC development. The liver is constantly exposed to microbial products from the enteric microflora, such as endotoxin, which activates proinflammatory signaling pathways and might contribute to the development of liver cancer as it has been previously demonstrated for liver cirrhosis [43]. Zhang HL et al. (2012) demonstrated that already the induction of such a dysbiosis is sufficient to promote hepatocarcinogenesis by enhanced portal lipopolysaccharide (LPS) levels [39]. Interestingly, in the above-mentioned study of Dapito DH and colleagues, chronic treatment of mice with a low, nontoxic dose of LPS during DEN/CCl4-induced hepatocarcinogenesis led to a significant increase in tumor number, tumor size in liver compared with control animals, thus further arguing for a direct influence of gut microbiota on hepatocarcinogenesis [42].

LPS connects to toll-like receptors type 4 (TLR4), which are present on hepatocytes, Kupffer and stellate cells, causing the release of pro-inflammatory cytokines and activation of proliferative and anti-apoptotic signals. LPS level in plasma is associated with excess intestinal bacteria growth, changes in microbiota composition and increased intestinal permeability [44]. The critical role in the maintenance and progression of the inflammatory component in the pathogenesis of hepatic injury is played by the translocation of endotoxins obtained from GM into the circulatory system with an increase in TLR4 expression, which leads to the activation of pro-inflammatory cytokines (tumor necrosis factor-alpha (TNF-a) and interleukin (IL)-6 [45], thus causing systemic inflammation. Inflammasoms, as a multi-protein oligomeric complex containing leucine and nucleotide binding domains, are responsible for the activation of the inflammatory response, controlling the splitting of pro-inflammatory cytokines. It was shown that dysbiosis leads to increased TNF-a expression [46]. The increased activation and production of TLR4 and pro-inflammatory cytokines in dysbiosis may also lead to the recruitment and activation of hepatic immune cells, and the pro-inflammatory transmission of TLR4 signals is involved in sensitizing cells to the profibrotic signaling pathways and thus leads to liver fibrosis [43] contributing to the progression of liver disease [46]. Kupffer cells (KC), as hepatic immunological cells, are critical components of the innate immune system located in the sinusoidal vascular space [47].

KC can be activated by a variety of endogenous and exogenous stimuli, including endotoxins in the impairment of GM [47]. KC activation triggers the production of inflammatory cytokines such as TNF- $\alpha$ as well as active oxygen forms [47], which may also lead to tissue damage, including liver damage. Bile acids (BAs) play a crucial role in the development and progression of liver diseases, including HCC. BAs are metabolites of cholesterol (CS) and perform several essential functions: emulsification of fats, elimination of cholesterol out of the body, detergent properties, impact on intestinal motility, etc. Bile acids synthesized from cholesterol in the liver are necessary for metabolism of cholesterol and digestion of lipids [48]. Bile acids are deposited in the gallbladder and excreted into the duodenum during digestion [49]. More than 95\% of bile acids are reabsorbed in the terminal iliac and transported back to the liver through the portal vein. Bile acids contribute to the absorption of dietary fats, cholesterol, and fat-soluble vitamins [49]. In addition, bile acids also function as signal molecules that affect physiological processes [49], which include regulation of glucose and lipid metabolism through activation of the farnesoid X-receptor (FXR) and binding to the G-protein of the bile acid receptor [50-52]. Bile acids may also affect the gut microbiota, as they are directly related to the integrity of the intestinal mucosa and the synthesis of antibacterial peptides [53]. When bile acids bind to FXR, antimicrobial peptides such as angiogenin 1 are produced. These peptides can inhibit excess gut microbiota growth by increasing the potential of intestinal epithelial cells to prevent bacterial uptake, improving intestinal barrier function [53]. Gut microbiota in turn may affect the size and composition of the bile acid complex by converting primary bile acids to secondary ones $[54,55]$. This may subsequently change the metabolism of lipids and glucose, especially in NAFLD-predisposed people $[54,55]$. Another mechanism through which gut microbiota can contribute to liver disease is the production of short-chain fatty acids (SCFAs).

Gut microbiota splits non-digestible carbohydrates, releasing SCFAs in the human intestine [56]. The main SCFAs are acetate, propionate, and butyrate, which are metabolized by muscles, liver, and epithelium as such [56]. Studies on the role of SCFAs mainly focus on butyrate, the main energy source for colonocytes, which improves the barrier function of the large intestine [56] and therefore has a positive effect on intestinal permeability. Butyrate has been shown to improve intestinal barrier by induction of dense compound proteins and mucin mucin type 2 [57-59] and enhanced expression of claudin-1 [60]. Butyrate can induce apoptosis in the liver and inhibit cell proliferation in hepatocytes, suppressing the expression of type 1 sirtuin, while increasing the expression of miR22 , as a tumor suppressor [61]. In other words, butyrate can inhibit liver cancer cells. It has also been shown that butyrate increases the feeling of satiety, reduces food intake, and delays gastric emptying 
by activating free fatty acid receptors type 2 and 3 [62]. Normal body weight and glucose homeostasis were more often found in mice with a deficit of free fatty acid receptors of type 2 and 3 . Stimulation of intestinal hormones and inhibition of food ingestion by butyrate and propionate may represent a new mechanism by which gut microbiota regulates host metabolism. Finally, butyrate can also affect inflammation. Studies have shown that butyrate in the intestinal tract binds and activates the gamma receptor activated by the proliferator peroxis (PPAR-y), which counteracts the transduction of nuclear factor-kappa B (NF-kB), thus causing an anti-inflammatory effect [63]. Therefore, the presence or excess of gut microbiota-produced butyrate can affect the pathogenesis of liver diseases through several mechanisms [63].

Choline deficiency also plays the most important role in liver injury with gut micriobiota imbalance. Choline is an essential nutrient and phospholipid component of the cell membrane [64]. There are several mechanisms through which choline deficiency can affect the liver, including [64] the reduction of very lowdensity lipoprotein formation (VLDL), dysfunction, mitochondrion, and endoplasmic reticulum stress [64,65]. Phosphatidylcholine, which is a phospholipid, is a key component of the VLDL shell. Choline deficiency caused by a diet or gut microbiota metabolism disorder leads to a decrease formation of VLDL and the export of triglycerides from the liver, resulting in fatty hepatosis. Choline deficiency reduces the concentration of phosphatidyl ethanolamine and phosphatidylcholine in mitochondrial membrane, leads to a decrease in membrane potential, which in turn causes oxidative damage [64] of cell membrane. Gut microbiota can help reduce the bioavailability of choline [66] contained in eggs, milk, and red meat. This in turn increases the conversion of choline into trimethylamine (TMA) [67], which is absorbed into the blood, increasing the risk of cardiovascular disease [67]. TMA reaching the liver is further metabolized by flavin-containing monoxygenases of types 1 and 3 to form trimethylamine-N-oxide (TMAO) [67-69]. This can lead to an increase in the accumulation of hepatic triglycerides, as TMAO inhibits key enzymes and limits the enterohepatic circulation of bile acids [70-72]. In such a way the formation of choline deficiency either through diet or through conversion of choline into TMA in case of GM metabolism disorder may lead to liver injury through accumulation of fat in it. The next factor impairing the enterohepatic relationship is alcohol abuse. Studies in the United States and Italy have shown that alcohol is the main cause of HCC (ranging from $32 \%$ to $45 \%$ of HCC). The EPIC (The European Prospective Investigation into Cancer and Nutrition) multi-centre cohort study from 1992 to 2000 selected about 520,000 randomly selected men and women aged 35-70 from 10 European countries [73].

Among men and women, 33\% (11\% - 54\%) and 18\% (3\% - 38\%) of the total number of HCC was due to past and present alcohol consumption [74]. The causal link between alcohol consumption and HCC development may be due to direct (hepotoxic) and indirect factors (cirrhosis development) [75]. Case-control studies from different countries report that chronic ethanol consumption is associated with approximately double increase in the odd ratio of HCC development. Obesity and alcohol synergistically contribute to the progression of ALD and development of HCC, although data in this regard are still contradictory [76-80]. Alcohol consumption causes significant changes in the quality and quantity of GM, changes in the mucous membrane, and increased intestinal permeability, which leads to endotoxemia [81] due to lipoteichoic acid, flagellin, bacterial hypomethylated DNA, and other intestinal toxins. Intestinal hyperpermeability due to alcohol abuse leads to a higher concentration of LPS in the portal bloodstream, which binds to TLR4, releases pro-inflammatory cytokines, produces active oxygen forms, and activates oxidative stress. In addition, regardless of alcohol consumption, ethanol is produced endogenously in small amounts under normal intermediate metabolism and in the gastrointestinal tract through the formation of microbes. The concentrations caused by this process in human venous blood are approximately 0-50 $\mu \mathrm{m}$ [82]. Dense compounds of the intestinal epithelium are impaired by acetaldehyde by increasing intestinal permeability to endotoxins. All these events can cause the activation of macrophages, Kupffer and stellate cells of the liver, supporting inflammation and fibrosis in the liver [83,84].

Impairment of the intestinal barrier may also cause the bacteria to move into the mesenteric lymph nodes, which further confirms systemic inflammation [85]. Formation of a strong inflammatory environment caused by direct exposure of alcohol to nonparenchymatous cells of the liver or indirectly fed by intestinal hyperpermeability is a condition that enhances the process of liver injury and regeneration and certainly favors the formation and reproduction of liver tumor foci [86]. The main causes of alcoholrelated oncogenesis are not yet fully understood though, various factors have been suggested that play a part: localized effects of alcohol, induction of cytochrome P4502E1 (CYP2E1) (conversion of various xenobiotics), acetaldehyde (isoenzyme polymorphism), malnutrition, interaction with retinoids, changes in methylation levels, immunological surveillance, and angiogenesis [87-89]. It was shown that the concentration of CYP2E1 in the liver may be associated with the formation of hydroxyethyl radical and, consequently, with lipid peroxidation. Lipid peroxidation triggers the production of 4-hydroxynonenal, which can bind to the purine and pyrimidine bases of DNA, thus forming carcinogenic exocyclic etheno-DNA adducts. It has been shown that there is a significant relationship between the induction of CYP2E1 and the appearance of exocyclic etheno-DNA-adducts in hepatocytes [88,90]. As such, the abuse of ethanol causes qualitative and quantitative changes in the taxonomic composition of the intestinal flora, inflammation 
of the mucous membrane and impairment of the intestinal barrier, with subsequent translocation of viable pathogenic bacteria, gram-negative microbial products and pro-inflammatory lumen metabolites into the bloodstream, which additionally confirms alcohol-induced liver injury.

Obesity and high fat diet have been identified as major risk factors for HCC [91,92]. In a prospective study of more than 900,000 adult American patients (404,576 males and 49,547 females) it was found that overweight and obesity have been accompanied by a significant increase in esophagus cancer, colorectal cancer, liver cancer, gall bladder cancer, pancreatic and kidney cancer mortality rates. For liver carcinoma, the odds ratio was 4.52 for men and 1.68 for women [93]. Yoshimoto S and colleagues demonstrated that administration of antibiotics and gut sterilization lead to a significant decrease in HCC development in an obesity related model of hepatocarcinogenesis in mice [36]. The authors demonstrated that obesity induced alterations of gut microbiota lead to elevated levels of deoxycholic acid (DCA), a gut bacterial metabolite, which induces the secretion of various inflammatory and procarcinogenic factors in the liver and thus facilitated HCC development. In this model eradication or modulation of the gut microflora blocked the DCA-inflammation-HCC axis, thus preventing obesity related liver tumors [36].

\section{Conclusion}

Thus, the data of the literature review indicate that disorders of enterohepatic relationships due to changes in gut microbiota with excess bacterial growth, intestinal barrier hyperpermeability increases the content of LPS, which when combined with TLT4receptors on hepatocytes, Kupffer and stellate cells, cause the activation of pro-inflammatory cytokines, production of activated forms of oxygen, form a pro-inflammatory microenvironment of the liver that promotes the development of HCC. In the light of a lack of pharmacopreventive strategies and limited chemotherapeutic options for treatment of liver cancer, the therapeutic modulation study of the gut microflora, such as Fecal Microbiota Transplantation (FMT) and Probiotic Interventions, requires the continuation of experimental and clinical studies to prevent the progression from chronic hepatitis to liver cirrhosis and HCC.

\section{References}

1. Singal AG, Lompertico P, Nahon P (2020) Epidemiology and surveillance for hepatocellular carcinoma: New trends. J of Hepatiol 72(2): 250-261.

2. Tao X, Wang N, Qin W (2015) Gut Microbiota and Hepatocellular Carcinoma. Gastrointest Tumors 2(1): 33-40.

3. Chang MH, You SL, Chen CJ, Liu CJ, Lai MW et al. (2016) Long-term effects of hepatitis B immunization of infants in preventing liver cancer Gastroenterology 151(3): 472-480.

4. (2012) World Gastroenterology Organisation Global Guidelines Nonalcoholic fatty liver disease and nonalcoholic steatohepatitis.
5. Sabirov I (2020) Practical aspects of the use of ezetimibe in non-alcoholic fatty liver disease. The Scientific Heritage 2(47): 50-57.

6. Mittal S, El Serag HB, Sada YH, Kanwal F, Zhigang Duan, et al. (2016) Hepatocellular carcinoma in the absence of cirrhosis in United States veterans is associated with nonalcoholic fatty liver disease. Clin Gastroenterol Hepatol 14(1): 124-131.

7. Stine JG, Wentworth BJ, Zimmet A, Rinella M, Rohit Loomba, et al. (2018) Systematic review with meta-analysis: risk of hepatocellular carcinoma in non-alcoholic steatohepatitis without cirrhosis compared to other liver diseases. Aliment Pharmacol Ther 48(7): 696-703.

8. Akinyemiju T, Abera S, Ahmed M, Alam N, Mulubirhan Assefa Alemayohu, et al. (2017) The Burden of Primary Liver Cancer and Underlying Etiologies from 1990 to 2015 at the Global, Regional, and National Level: Results From the Global Burden of Disease Study 2015. JAMA Oncol 3(12): 1683-1691.

9. Hsu IC, Metcalf RA, Sun T, Welsh JA, N J Wang et al. (1991) Mutational hotspot in the p53 gene in human hepatocellular carcinomas. Nature 350(6317): 427-428.

10. Lee YC, Cohet C, Yang YC, Stayner L, Mia Hashibe, et al. (2009) Metaanalysis of epidemiologic studies on cigarette smoking and liver cancer Int J Epidemiol 38(6): 1497-1511.

11. (2018) European Association for the Study of the Liver. European Association for the Study of the Liver. EASL Clinical Practice Guidelines: Management of hepatocellular carcinoma. J Hepatol 69(1): 182-236.

12. Tseng $\mathrm{CH}$ (2018) Metformin and risk of hepatocellular carcinoma in patients with type 2 diabetes. Liver Int 38(11): 2018-2027.

13. Simon TG, Ma Y, Ludvigsson JF, Chong D, Edward L Giovannucci, et al. (2018) Association Between Aspirin Use and Risk of Hepatocellular Carcinoma. JAMA Oncol 4(12): 1683-1690.

14. Jemal A, Bray F, Center MM, Ferlay J, Elizabeth Ward, et al. (2011) Global cancer statistics. CA: Cancer J Clin 61(2): 69-90.

15. Stärkel P, Schnabl B (2016) Bidirectional Communication between Liver and Gut during Alcoholic Liver Disease. Semin Liver Dis 36(4): 331-339.

16. Wang X, Zhang A, Miao J, Sun H, Guang li Yan, et al. (2018) Gut microbiota as important modulator of metabolism in health and disease. RSC Advances 8(74): 42380-42389.

17. Montalto M, D’Onofrio F, Gallo A, Cazzato A, G.Gasbarrini, et al. (2009) Intestinal microbiota and its functions. Digestive Liver Disease Supplements 3(2): 30-34.

18. Qin J, Li R, Raes J, Manimozhiyan Arumugam, Kristoffer Solvsten Burgdorf, et al. (2010) A human gut microbial gene catalogue established by metagenomic sequencing. Nature 464(7285): 59-65.

19. Sharkey KA, Beck PL, McKay DM (2018) Neuroimmunophysiology of the gut: advances and emerging concepts focusing on the epithelium. Nat Rev Gastroenterol Hepatol 15(12): 765-784.

20. Sharpton SR, Ajmera V, Loomba R (2019) Emerging role of the gut microbiome in nonalcoholic fatty liver disease: from composition to function. Clin Gastroenterol Hepatol 17(2): 296-306.

21. Van Leeuwen PA, Boermeester MA, Houdijk AP, Ferwerda CC, M A Cuesta, et al. (1994) Clinical significance of translocation. Gut 35(1): 28-34.

22. Jirillo E, Caccavo D, Magrone T, Piccigallo E, L Amati, et al. (2002) The role of the liver in the response to LPS: experimental and clinical findings. J Endotoxin Res 8(5): 319-327.

23. Rooks MG, Garrett WS (2016) Gut microbiota, metabolites and host immunity. Nat Rev Immunol 16(6): 341-352.

24. Thaiss CA, Zmora N, Levy M, Elinav E (2016) The microbiome and innate immunity. Nature 535(7610): 65-74. 
25. Dore J, Simren M, Buttle L, Guarner F (2013) Hot topics in gut microbiota. United European Gastroenterol J 1(5): 311-318.

26. Simren M, Barbara G, Flint HJ, Spiegel BM, Robin C Spiller, et al. (2013) Intestinal microbiota in functional bowel disorders: a Rome foundation report. Gut 62(1): 159-176.

27. Ahmed I, Roy BC, Khan SA, Septer S, Shahid Umar (2016) Microbiome, metabolome and inflammatory bowel disease. Microorganisms 4(2): 20

28. Cani PD, Delzenne NM (2009) The role of the gut microbiota in energy metabolism and metabolic disease. Curr Pharm Des 15(3): 1546-1558.

29. Duseja A, Chawla YK (2014) Obesity and NAFLD: The role of bacteria and microbiota. Clin Liver Dis 18(1): 59-71.

30. Fox JG, Feng Y, Theve EJ, J L A Fiala, A L Doernte, et al. (2010) Gut microbes define liver cancer risk in mice exposed to chemical and viral transgenic hepatocarcinogens. Gut 59(1): 88-97.

31. Rogers AB (2011) Distance burning: how gut microbes promote extraintestinal cancers. Gut Microbes 2(1): 52-57.

32. Huang Y, Fan XG, Wang ZM, Zhou JH, XF Tian, et al. (2004) Identification of helicobacter species in human liver samples from patients with primary hepatocellular carcinoma. J Clin Pathol 57(12): 1273-1277.

33. Ward JM, Anver MR, Haines DC, Benveniste RE (1994) Chronic active hepatitis in mice caused by Helicobacter hepaticus. Am J Pathol 145(4): 959-968.

34. Rocha M, Avenaud P, Ménard A, Le Bail B, C Balabaud, et al. (2005) Association of Helicobacter species with hepatitis C cirrhosis with or without hepatocellular carcinoma. Gut 54(3): 396-401.

35. Krüttgen A, Horz HP, Weber Heynemann J, Vucur M, Christian Trautwein, et al. (2012) Study on the association of Helicobacter species with viral hepatitis-induced hepatocellular carcinoma. Gut Microbes 3(3): 228233.

36. Yoshimoto S, Loo TM, Atarashi K, Kanda H, Seidai Sato, et al. (2013) Obesity-induced gut microbial metabolite promotes liver cancer through senescence secretome. Nature. 499(7456): 97-101.

37. Xie G, Wang X, Liu P, Wei R, Wenlian Chen, et al. (2016) Distinctly altered gut microbiota in the progression of liver disease. Oncotarget $7(15)$ : $19355-19366$.

38. Grąt M, Krasnodębski M, Patkowski W, Wronka K, Łukasz Masior, et al. (2016) Relevance of Pre-Transplant $\alpha$-fetoprotein Dynamics in Liver Transplantation for Hepatocellular Cancer. Ann Transplant 21: 115-124.

39. Zhang HL, Yu LX, Yang W, Tang L, Yan Lin, et al. (2012) Profound impact of gut homeostasis on chemically-induced pro-tumorigenic inflammation and hepatocarcinogenesis in rats. J Hepatol 57(4): 803-812.

40. Karin M (2018) New insights into the pathogenesis and treatment of non-viral hepatocellular carcinoma: a balancing act between immunosuppression and immunosurveillance. Precision Clinical Medicine 1(1): 21-28.

41. Yu LX, Yan HX, Liu Q Yang W, Hong Ping Wu, et al. (2010) Endotoxin accumulation prevents carcinogen-induced apoptosis and promotes liver tumorigenesis in rodents. Hepatology 52(4): 1322-1333.

42. Dapito DH, Mencin A, Gwak GY, Pradere JP, Myoung-Kuk Jang, et al. (2012) Promotion of hepatocellular carcinoma by the intestinal microbiota and TLR4. Cancer Cell 21(4): 504-516.

43. Seki E, De Minicis S, Osterreicher CH, Yosuke Osawa, et al. (2007) TLR4 enhances TGF-beta signaling and hepatic fibrosis. Nat Med 13(11): 1324-1332.

44. Douhara A, Moriya K, Yoshiji H, Noguchi R, Tadashi Namisaki, et al. (2015) Reduction of endotoxin attenuates liver fibrosis through suppression of hepatic stellate cell activation and remission of intestinal permeability in a rat non-alcoholic steatohepatitis model. Mol Med Rep 11(3): 16931700 .
45. Jiang W, Wu N, Wang X, Chi Y, Yuanyuan Zhang, et al. (2015) Dysbiosis gut microbiota associated with inflammation and impaired mucosal immune function in intestine of humans with non-alcoholic fatty liver disease. Sci Rep 5: 8096.

46. Wong VW, Tse CH, Lam TT, Wong GL, Angel Mei-Ling Chim, et al (2013) Molecular characterization of the fecal microbiota in patients with nonalcoholic steatohepatitis-a longitudinal study. PLoS One 8(4): e62885.

47. Baffy G (2009) Kupffer cells in non-alcoholic fatty liver disease: the emerging view. J Hepatol 51(1): 212-223.

48. Staley C, Weingarden AR, Khoruts A, Sadowsky MJ (2017) Interaction of gut microbiota with bile acid metabolism and its influence on disease states. Appl Microbiol Biotechnol 101(1): 47-64.

49. Long SL, Gahan CGM, Joyce SA (2017) Interactions between gut bacteria and bile in health and disease. Mol Aspects Med 56: 54-65.

50. Sinal CJ, Tohkin M, Miyata M, Ward JM, G Lambert, et al. (2000) Targeted disruption of the nuclear receptor FXR/BAR impairs bile acid and lipid homeostasis. Cell 102(6): 731-744.

51. Hylemon PB, Zhou H, Pandak WM, Ren S, Gregorio Gil, et al. (2009) Bile acids as regulatory molecules. J Lipid Res 50(8): 1509-1520.

52. Copple BL, Li T (2016) Pharmacology of bile acid receptors: Evolution of bile acids from simple detergents to complex signaling molecules. Pharmacol Res 104: 9-21.

53. Parséus A, Sommer N, Sommer F, Caesar R, Antonio Molinaro, et al (2017) Microbiota-induced obesity requires farnesoid X receptor. Gut 66(3): 429-437.

54. Mouzaki M, Wang AY, Bandsma R, Comelli EM, Bianca M Arendt, et al. (2016) Bile Acids and Dysbiosis in Non-Alcoholic Fatty Liver Disease. PLoS One 11(5): e0151829.

55. Ridlon JM, Kang DJ, Hylemon PB, Bajaj JS (2014) Bile acids and the gut microbiome. Curr Opin Gastroenterol 30(3): 332-338.

56. Mouzaki M, Wang AY, Bandsma R, Comelli EM, Bianca M Arendt, et al (2016) Bile Acids and Dysbiosis in Non-Alcoholic Fatty Liver Disease. PLoS One 11(5): e0151829.

57. Hamer HM, Jonkers D, Venema K, Vanhoutvin S, F J Troost, et al. (2008) Review article: the role of butyrate on colonic function. Aliment Pharmacol Ther 27(2): 104-119.

58. Gaudier E, Rival M, Buisine MP, Robineau I, C Hoebler (2009) Butyrate enemas upregulate Muc genes expression but decrease adherent mucus thickness in mice colon. Physiol Res 58(1): 111-119.

59. Willemsen LE, Koetsier MA, van Deventer SJ, van Tol EA (2003) Short chain fatty acids stimulate epithelial mucin 2 expression through differential effects on prostaglandin $E(1)$ and $E(2)$ production by intestinal myofibroblasts. Gut 52(10): 1442-1447.

60. Augenlicht L, Shi L, Mariadason J, Laboisse C, Velcich A (2003) Repression of MUC2 gene expression by butyrate, a physiological regulator of intestinal cell maturation. Oncogene 22(32): 4983-4992.

61. Wang HB, Wang PY, Wang X, Wan YL, Yu Cun Liu (2012) Butyrate enhances intestinal epithelial barrier function via up-regulation of tight junction protein Claudin-1 transcription. Dig Dis Sci 57(12): 3126-3135.

62. Pant K, Yadav AK, Gupta P, Islam R, Anoop Saraya, et al. (2017) Butyrate induces ROS-mediated apoptosis by modulating miR-22/SIRT-1 pathway in hepatic cancer cells. Redox Biol 12: 340-349.

63. Lin HV, Frassetto A, Kowalik EJ Jr, Nawrock A, Mofei M Lu, et al. (2012) Butyrate and propionate protect against diet-induced obesity and regulate gut hormones via free fatty acid receptor 3-independent mechanisms. PLoS One 7(4): e35240. 
64. Kinoshita M, Suzuki Y, Saito Y (2002) Butyrate reduces colonic paracellular permeability by enhancing PPARgamma activation. Biochem Biophys Res Commun 293(2): 827-831.

65. Corbin KD, Zeisel SH (2012) Choline metabolism provides novel insights into nonalcoholic fatty liver disease and its progression. Curr Opin Gastroenterol 28(2): 159-165.

66. Zeisel SH, da Costa KA (2009) Choline: an essential nutrient for public health. Nutr Rev 67(11): 615-623.

67. Craciun S, Balskus EP (2012) Microbial conversion of choline to trimethylamine requires a glycyl radical enzyme. Proc Natl Acad Sci USA 109(52): 21307-21312.

68. Wang Z, Klipfell E, Bennett BJ, Koeth R, Bruce S Levison, et al. (2011) Gut flora metabolism of phosphatidylcholine promotes cardiovascular disease. Nature 472(7341): 57-63.

69. Howitt MR, Garrett WS (2012) A complex microworld in the gut: gut microbiota and cardiovascular disease connectivity. Nat Med 18(8): 1188-1189.

70. Shih DM, Wang Z, Lee R, Meng Y, Nam Che, et al. (2015) Flavin containing monooxygenase- 3 exerts broad effects on glucose and lipid metabolism and atherosclerosis. J Lipid Res 56(1): 22-37.

71. Chen YM, Liu Y, Zhou RF, Chen XL, Cheng Wang, et al. (2016) Associations of gut-flora-dependent metabolite trimethylamine- $\mathrm{N}$-oxide, betaine and choline with non-alcoholic fatty liver disease in adults. Sci Rep 6: 19076

72. Sherriff JL, O'Sullivan TA, Properzi C, Oddo JL, Leon A Adams (2016) Choline, its role in nonalcoholic fatty liver disease, and the case for human and bacterial genes. Adv Nutr 7(1): 5-13.

73. Spencer MD, Hamp TJ, Reid RW, Fischer LM, Steven H Zeisel, et al. (2011) Association between composition of the human gastrointestinal microbiome and development of fatty liver with choline deficiency. Gastroenterology 140(3): 976-986

74. Riboli E, Hunt KJ, Slimani N, Pietro Ferrari, T Norat, et al. (2002) European Prospective Investigation into Cancer and Nutrition (EPIC) study populations and data collection. Public Health Nutr. 5(6B):11131124 .

75. Schütze M, Boeing H, Pischon T, Rehm J, Tara Kehoe, et al. (2011) Alcohol attributable burden of incidence of cancer in eight European countries based on results from prospective cohort study. BMJ. 342: d1584.

76. Donato F, Tagger A, Gelatti U, Parrinello G, P Boffetta, et al. (2002) Alcohol and hepatocellular carcinoma: the effect of lifetime intake and hepatitis virus infections in men and women. Am J Epidemiol 155(4): 323-331.

77. Crombie IK, Cunningham KB, Irvine L, Williams B, Falko F Sniehotta, et al. (2017) Modifying Alcohol Consumption to Reduce Obesity (MACRO): development and feasibility trial of a complex community-based intervention for men. Health Technol Assess 21(19): 1-150.

78. Loomba R, Yang HI, Su J, Brenner D, Elizabeth Barrett Connor, et al (2013) Synergism between obesity and alcohol in increasing the risk of hepatocellular carcinoma: a prospective cohort study. Am J Epidemiol 177(4): 333-342.
79. Hart CL, Morrison DS, Batty GD, Mitchell RJ, George Davey Smith (2010) Effect of body mass index and alcohol consumption on liver disease: analysis of data from two prospective cohort studies. BMJ 340: c1240.

80. Vimaleswaran KS, Cavadino A, Verweij N, Nolte I, Irene Mateo Leach, et al. (2015) Interactions between uncoupling protein 2 gene polymorphisms, obesity and alcohol intake on liver function: a large meta-analysed population-based study. Eur J Endocrinol 173(6): 863-872.

81. Huo TI, Wu JC, Lee SD (2005) Are alcohol, tobacco and obesity genuine risk factors for hepatocellular carcinoma?. J Hepatol 42(6): 941-942.

82. Bode C, Bode JC (2003) Effect of alcohol consumption on the gut. Best Pract Res Clin Gastroenterol 17(4): 575-592.

83. Welzel TM, Graubard BI, Quraishi S, Zeuzem S, Austin G Duffy, et al. (2013) Population-attributable fractions of risk factors for hepatocellular carcinoma in the United States. Am J Gastroenterol 108(8): 1314-1321.

84. Bala S, Marcos M, Kodys K, Csak T, Donna Catalano, et al. (2011) Upregulation of microRNA-155 in macrophages contributes to increased tumor necrosis factor - alpha (TNF-alpha) production via increased mRNA half-life in alcoholic liver disease. J Biol Chem 286(2): 1436-1444.

85. Hritz I, Mandrekar P, Velayudham A, Catalano D, Angela Dolganiuc, et al. (2008) The critical role of toll-like receptor (TLR) 4 in alcoholic liver disease is independent of the common TLR adapter MyD88. Hepatology 48(4): 1224-1231.

86. Leclercq S, Matamoros S, Cani PD, Neyrinck AM, François Jamar, et al (2014) Intestinal permeability, gut-bacterial dysbiosis, and behavioral markers of alcohol-dependence severity. Proc Natl Acad Sci USA 111(42): E4485-E4493.

87. Nagy LE (2015) The Role of Innate Immunity in Alcoholic Liver Disease. Alcohol Res 37(2): 237-250.

88. Lachenmeier DW, Sohnius EM (2008) The role of acetaldehyde outside ethanol metabolism in the carcinogenicity of alcoholic beverages: evidence from a large chemical survey. Food Chem Toxicol 46(8): 29032911

89. Seitz HK, Stickel F, Homann N (2004) Pathogenetic mechanisms of upper aerodigestive tract cancer in alcoholics. Int J Cancer 108(4): 483-487.

90. Seitz HK, Stickel F (2007) Molecular mechanisms of alcohol-mediated carcinogenesis. Nat Rev Cancer 7(8): 599-612.

91. Ley RE, Turnbaugh PJ, Klein S, Gordon JI (2006) Microbial ecology: human gut microbes associated with obesity. Nature 444(7122): 10221023.

92. Adami HO, Trichopoulos D (2003) Obesity and mortality from cancer. N Engl J Med 348(17): 1623-1624.

93. Calle EE, Rodriguez C, Walker Thurmond K, Thun MJ (2003) Overweight, obesity, and mortality from cancer in a prospectively studied cohort of U.S. adults. N Engl J Med 348(17): 1625-1638. 